\title{
Article
}

\section{Fuzzy Entropy-Based Spatial Hotspot Reliability}

\author{
Ferdinando Di Martino ${ }^{1,2, *(\mathbb{D})}$ and Salvatore Sessa ${ }^{1,2}$ (D) \\ 1 Dipartimento di Architettura, Università degli Studi di Napoli Federico II, Via Toledo 402, 80134 Napoli, Italy; \\ sessa@unina.it \\ 2 Centro Interdipartimentale di Ricerca in Urbanistica Alberto Calza Bini, Università degli Studi di Napoli \\ Federico II, Via Toledo 402, 80134 Napoli, Italy \\ * Correspondence: fdimarti@unina.it
}

check for updates

Citation: Di Martino, F.; Sessa, S. Fuzzy Entropy-Based Spatial Hotspot Reliability. Entropy 2021, 23, 531. https://doi.org/10.3390/e23050531

Academic Editor: Pavel Sevastjanov

Received: 8 April 2021

Accepted: 22 April 2021

Published: 26 April 2021

Publisher's Note: MDPI stays neutral with regard to jurisdictional claims in published maps and institutional affiliations.

Copyright: (C) 2021 by the authors Licensee MDPI, Basel, Switzerland. This article is an open access article distributed under the terms and conditions of the Creative Commons Attribution (CC BY) license (https:// creativecommons.org/licenses/by/ $4.0 /)$.

\begin{abstract}
Cluster techniques are used in hotspot spatial analysis to detect hotspots as areas on the map; an extension of the Fuzzy C-means that the clustering algorithm has been applied to locate hotspots on the map as circular areas; it represents a good trade-off between the accuracy in the detection of the hotspot shape and the computational complexity. However, this method does not measure the reliability of the detected hotspots and therefore does not allow us to evaluate how reliable the identification of a hotspot of a circular area corresponding to the detected cluster is; a measure of the reliability of hotspots is crucial for the decision maker to assess the need for action on the area circumscribed by the hotspots. We propose a method based on the use of De Luca and Termini's Fuzzy Entropy that uses this extension of the Fuzzy C-means algorithm and measures the reliability of detected hotspots. We test our method in a disease analysis problem in which hotspots corresponding to areas where most oto-laryngo-pharyngeal patients reside, within a geographical area constituted by the province of Naples, Italy, are detected as circular areas. The results show a dependency between the reliability and fluctuation of the values of the degrees of belonging to the hotspots.
\end{abstract}

Keywords: hotspots; fuzzy clustering; FCM; EFCM; fuzzy entropy; reliability

\section{Introduction}

Hotspot detection is an emerging spatial analysis feature that allows for the detection of areas in which events representing a certain phenomenon are present with greater insistence (hotspots) and follows their spatial distribution and displacement over time. Cluster techniques are proposed by various researchers to locate hotspots in the study area for many problems. For example, in crime analysis, it is used specifically to locate as hotspots the areas with greater presence and frequency of criminal events in city contexts; in disease analysis, it is used to evaluate the formation and displacement of disease strains over time; in monitoring problems of natural and environmental disasters, such as the monitoring of developments of fires in wooded areas in summer, it is applied to analyze where and with what frequency and intensity natural and malicious phenomena of fires develop.

Cluster algorithms are proposed by some authors to detect hotspots in various spatial analysis problems.

K-means [1] is applied by some authors to detect hotspots in crime analysis [2,3] and fire analysis [4,5]. K-medoids [6] is applied in disease analysis [7]. Fuzzy C-means (for short, FCM) [8-10] is applied to detect hotspots in crime analysis [11-14], road traffic crashes [15], and disease analysis [16]. Kernel density-based algorithms [17] are applied in crime analysis [18], soil pollution [19], and traffic accident analysis [20].

Kernel density algorithms have the advantage of detecting even hotspots of irregular geometric shape, but they are computationally more expensive than K-means and FCM; on the other hand, K-means and FCM detect only cluster centers and are less robust than the 
presence of noise in the data. Furthermore, in K-means and FCM, the number of clusters must be fixed in advance and validity measures must be used to evaluate what the suitable number of clusters might be.

In [21], a new hotspot detection technique is proposed, based on an extension of FCM, called Extended Fuzzy C-means (for short, EFCM) [22]. EFCM detect cluster as hyper-spheres in the space of the features; the number of clusters must not be set a priori as it is obtained through merging processes of the most similar clusters carried out during each iteration. In [21], the authors show that EFCM can approximate the shape of hotspots on the map and is robust with respect to the presence of noise and outliers. The EFCM hotspot detection method was applied in disease analysis $[23,24]$ and in earthquake disaster analysis [25].

One of the main needs in hotspot detection is to evaluate the reliability of the results by measuring how significant the detected hotspots are. EFCM detects circular hotspots on the map but does not give information about their reliability. This assessment is sometimes interpretative; it is left to the expert who assesses whether the analyzed event persists more frequently in the region where the hotspot was detected. An effective measure of reliability of a hotspot is critical to understanding how accurate the location of the area in which the analyzed phenomenon exists is, in order to monitor it and follow its movements over time. Currently, no hotspot detection method proposed in the literature allows us to measure the reliability of the detected hotspots; a quantitative assessment of the reliability of the detected hotspots is crucial because it would allow the decision maker to evaluate how accurate the geographic location and areal size of a hotspot can be.

In this research we propose a measure of the reliability of the hotspots detected by running the EFCM algorithm, in which the De Luca \& Termini fuzzy entropy $[26,27]$ is applied; the reliability of the hotspot is higher if the fuzzy entropy measured for the corresponding cluster is lower.

Recently, measures of fuzzy entropy of clustering in FCM have been proposed in [28,29]. Following [28], in this work each fuzzy cluster constitutes a fuzzy set in the domain of the data points and a measure of the fuzzy entropy is applied to each fuzzy cluster to evaluate its fuzziness.

If $H\left(A_{i}\right)$ is the fuzziness of the $i$ th cluster, normalized in the interval [0,1], we assign a reliability of the correspondent hotspot $A_{i}$ given by:

$$
R\left(A_{i}\right)=1-H\left(A_{i}\right)
$$

The reliability of $A_{i}$ is normalized in the interval [0,1]. It holds 1 when the fuzziness of $C_{i}$ is null (the cluster is a not null crisp set) and 0 when the fuzziness is maximum (all the data points belong to the cluster with a membership degree $\frac{1}{2} n$ ).

$\mathrm{We}^{\prime}$ ve implemented our method in a GIS-framework in which the EFCM-based hotspot detection algorithm was encapsulated. After executing EFCM, the detected hotspots are shown as circles on the map and the reliability of each hotspot is calculated as in (1). Finally, the hotspot reliability map is constructed.

In next section, the EFCM algorithm and the De Luca \& Termini Fuzzy Entropy are introduced. Our framework is described in Section 3. Section 4 show the results of our tests. Finally, considerations are included in Section 5.

\section{Preliminaries}

\subsection{The EFCM Algorithm}

The EFCM algorithm [22] is a variation of FCM in which the cluster prototypes are hyper-spheres in the space of the features; each cluster is characterized by a vector characterizing its center and its radius.

Let $X=\left\{x_{1}, \ldots, x_{N}\right\} \subset R^{n}$ be a set of $N$ data points in the $n$-dimensional space of the features $R^{n}$ where $x_{k}=\left(x_{k 1}, \ldots, x_{k n}\right)$. Let $V=\left\{v_{1}, \ldots, v_{C}\right\} \subset R^{n}$ be the set of centers of the $C$ clusters. Let $U$ be the $C \times N$ partition matrix where $u_{i k}$ is the membership degree of the $k$ th data point $x_{k}$ to the $j$ th cluster $v_{j}$. Let $r=\left\{r_{1}, \ldots, r_{C}\right\}$ be the set of radii of the $C$ clusters. 
EFCM minimize the following objective function:

$$
J(\boldsymbol{U}, \boldsymbol{V}, \boldsymbol{r})=\sum_{i=1}^{C} \sum_{j=1}^{N} u_{i j}^{m} \delta_{i j}^{2}
$$

where $m$ is the fuzzifier parameter and $\delta_{i j}$, interpreted as the distance between the $i$ th cluster and the $j$ th data point, is given by:

$$
\delta_{i j}=\max \left(0, d_{i j}-r_{i}\right)
$$

In (2) $d_{i j}$ is the Euclidean distance between the center of the $i$ th cluster and the $j$ th pattern and $r_{i}$ is the radius of the $i$ th cluster.

If $\boldsymbol{P}_{\boldsymbol{i}}$ is the covariance matrix of the $i$ th cluster:

$$
\boldsymbol{P}_{i}=\frac{\sum_{j=1}^{N} u_{i j}^{m}\left(x_{j}-v_{i}\right)\left(x_{j}-v_{i}\right)^{T}}{\sum_{j=1}^{N} u_{i j}^{m}}
$$

then the radius $r_{i}$ of the $i$ th cluster is given by:

$$
r_{i}=\sqrt{\operatorname{det}\left(P_{i}\right)^{1 / n}}
$$

Applying the Lagrange multiplier method to the (1) we obtain the solutions for $V$ and $U$ :

$$
\begin{gathered}
\boldsymbol{v}_{i}=\frac{\sum_{j=1}^{N} u_{i j}^{m} x_{j}}{\sum_{j=1}^{N} u_{i j}^{m}} i=1, \ldots, C \\
u_{i j}=\left\{\begin{array}{l}
\frac{1}{\sum_{k=1}^{C}\left(\frac{\delta_{i j}}{\delta_{k j}}\right)^{\frac{2}{(m-1)}}} \quad \text { if } \varphi_{j}=0 \\
\left\{\begin{array}{ll}
0 & \text { if } \delta_{i j}>0 \\
\frac{1}{\phi_{j}} & \text { if } \delta_{i j}=0
\end{array} \quad \text { if } \varphi_{j}>0\right.
\end{array} \quad i=1, \ldots, C, j=1, \ldots, N\right.
\end{gathered}
$$

where $\phi_{j}$ is the number of cluster whose distance from the $j$ th data point $\delta_{i j} i=1, \ldots, C$ is equal to 0 .

In [22], with the aim to ensure the separation between clusters, the radius of the $i$ th cluster calculated at the $t$ th iteration, $r_{i}^{(t)}$ is increased by a factor $\frac{\beta^{(t-1)}}{C^{(t-1)}}$, where $C^{(t-1)}$ is the number of clusters detected during the previous iteration and $\beta^{(t-1)}$ is the value calculated at the previous iteration of a parameter defined recursively, where $\beta^{(0)}=1$, $\beta^{(\tau)}=\min \left(C^{(\tau-1)}, \beta^{(\tau-1)}+1\right)$.

The optimal number of clusters is found merging at any iteration with the two most similar clusters, under some conditions.

The similarity between two clusters is measured by the following inclusion index:

$$
S_{i k}=\frac{\sum_{j=1}^{N} \min \left(u_{i j}, u_{k j}\right)}{\min \left(\sum_{j=1}^{N} u_{i j}, \sum_{j=1}^{N} u_{k j}\right)} \quad i=1, \ldots, C \quad j=1, \ldots, N
$$

where the similarity cluster matrix $\mathbf{S}$ is a symmetric matrix.

Let $\mathbf{S}^{(\mathrm{t})}$ be the similarity cluster matrix calculated at the $t$ th iteration and let $i^{*}$ and $k^{*}$ be the indices of the two most similar clusters; these two clusters are merged if their similarity is greater than an adaptive similarity threshold $\alpha^{(t)}=1 /\left(C^{(t)}-1\right)$, and the absolute difference $\left|S_{i^{*} k *}^{(t)}-S_{i^{*} k *}^{(t-1)}\right|$ is less than an error $\eta$. 
When two clusters are merged, the number of clusters is reduced by one unit and the parameter $\beta$ remains unchanged, otherwise, if $S_{i^{*} k *}^{(t)}>\alpha^{(t)}$, the parameter $\beta$ is increased, by setting $\beta^{(t)}=\min \left(C^{(t-1)}, \beta^{(t-1)}+1\right)$.

If the two most similar clusters are merged:

$$
\left\{\begin{array}{l}
u_{i * j}^{(t)}=u_{i * j}^{(t)}+u_{k * j}^{(t)} \quad \forall j \in\{1, \ldots, N\} \\
C^{(t)}=C^{(t-1)}-1 \\
\beta^{(t)}=\beta^{(t-1)}
\end{array}\right.
$$

otherwise:

$$
\left\{\begin{array}{l}
\text { if } S_{i^{*} k *}^{(t)}>\alpha^{(t)} \text { then } \beta^{(t)}=\min \left(C^{(t-1)}, \beta^{(t-1)}+1\right) \\
C^{(t)}=C^{(t-1)}
\end{array}\right.
$$

The EFCM algorithm (Algorithm 1) is described in the following pseudocode.

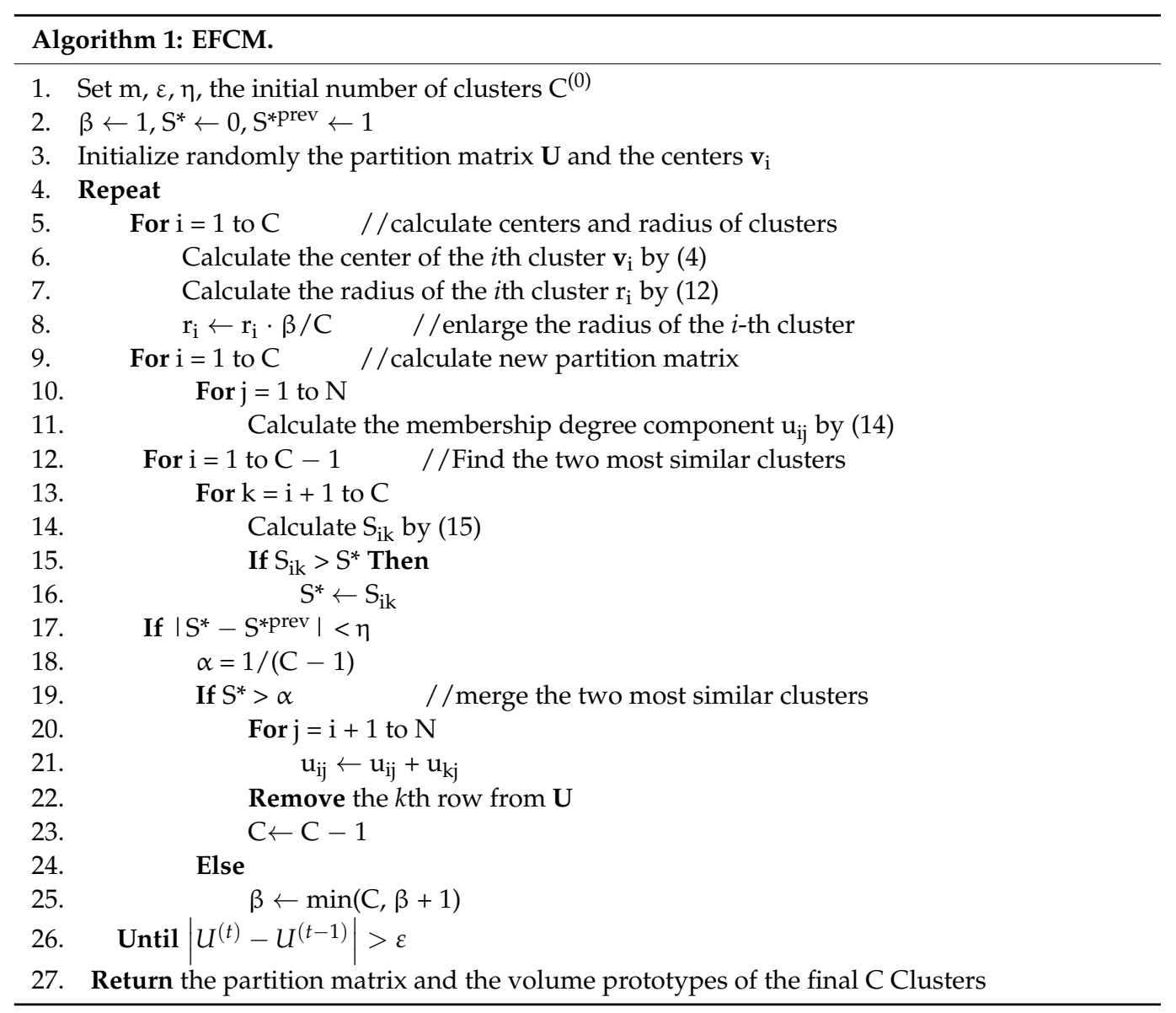

\subsection{De Luca \& Termini Fuzzy Entropy and the Measure of Fuzziness}

Let $F(X)=\{A: X \rightarrow[0,1]\}$ be the family of fuzzy sets defined on a universe of discourse $X$.

Let $h:[0,1] \rightarrow[0,1]$ be a continuous function called fuzzy entropy function. The following restrictions are required for the function $h$ :

1. $h(1)=0$

2. $h(\mathrm{u})=h(1-u)$

3. $h$ is monotonically increasing in in $\left[0, \frac{1}{2}\right]$

4. $h$ is monotonically decreasing in in $\left[\frac{1}{2}, 1\right]$

The fuzzy entropy function has a minimal value of 0 when $u$ is 0 or 1 , and a maximum value when $u=\frac{1}{2}$. 
De Luca and Termini in [26,27] propose the following fuzzy entropy function:

$$
h(u)=\left\{\begin{array}{cc}
0 & \text { if } u=0 \\
-u \cdot \log _{2}(u)-(1-u) \cdot \log _{2}(1-u) & \text { if } 0<u<1 \\
0 & \text { if } u=1
\end{array}\right.
$$

which has the maximum value 1 when $u=\frac{1}{2}$; it is called Shannon's function.

If $X=\left\{x_{i}, x_{2}, \ldots, x_{N}\right\}$ is a discrete set, we define the entropy measure of fuzziness of the fuzzy set $A$ as:

$$
H(A)=K \sum_{i=1}^{N} h\left(A\left(x_{i}\right)\right)
$$

where $K$ is a multiplicative constant. If $H(A)=0$, then for each element $x_{i}, i=1, \ldots, N$, $A\left(x_{i}\right)=0$ or $A\left(x_{i}\right)=1$ and A coincides with a subset of the set $\mathrm{X}$; if for each element $x_{i}$, $i=1, \ldots, N, A\left(x_{i}\right)=\frac{1}{2}$ and the fuzziness of $A$ is maximum.

If $A$ is a crisp set, its fuzziness is null and $H(A)=0$. The higher the fuzziness of a fuzzy set, the closer the mean membership degree to the fuzzy set of X's elements approaches $\frac{1}{2}$.

In [28], a fuzziness measure (12) is used to construct a new validity index, which is applied to evaluate the optimal number of clusters in FCM. If $A_{i}$ is the $i$ th fuzzy cluster where $i=1, \ldots, C$ considered as a fuzzy set and $\mathrm{u}_{\mathrm{ij}}$ is the membership degree of the $j$ th data point to the $i$ th cluster, the authors use the following fuzzy entropy measure of $A_{i}$ :

$$
H\left(A_{i}\right)=\frac{1}{N} \sum_{j=1}^{N} h\left(u_{i j}\right) \quad \mathrm{i}=1, \ldots, \mathrm{C}
$$

where $N$ is the number of data points and the De Luca \& Termini fuzzy entropy function (11) is used.

\section{The Proposed Framework}

We constructed a GIS-based framework in which EFCM is implemented to detect hotspots and the fuzzy entropy measure (13) is calculated to evaluate the reliability of the detected hotspots. The framework is schematized in Figure 1.

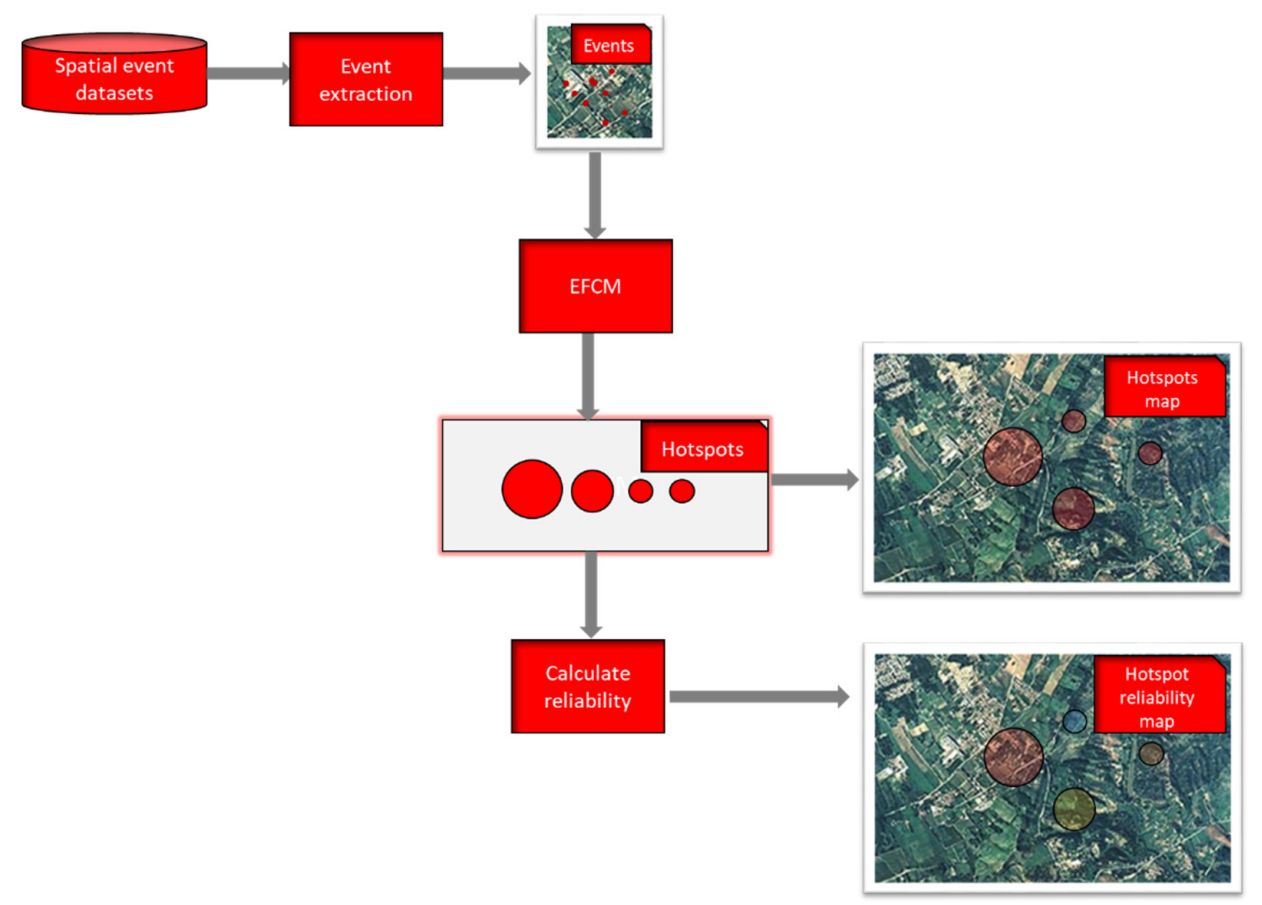

Figure 1. The proposed framework. 
The set of spatial events is extracted from the spatial event datasets. Each event is a data point given by its latitude and longitude coordinates. The event extraction functionality builds the set of data points by extracting the event data located in the study area and transforming them into a single system of geographic coordinates.

The set of spatial events is given by a set of $N$ events $X=\left\{x_{1}, \ldots, x_{N}\right\} R^{N}$. Each data point is given by a pair $x_{j}=\left(x_{j 1}, x_{j 2}\right), j=1, \ldots, N ; x_{j 1}$ and $x_{j 2}$ are the latitude and longitude coordinates where the event is located.

EFCM was executed to detect circles $C$ as clusters; each cluster is identified by its center $v_{j}=\left(v_{i 1}, v_{i 2}\right), j=1, \ldots, C$ and its radius $r_{i}$ and the component $u_{i j}$ of the $C \times N$ partition matrix $U$ gives the membership degree of the $j$ th data point to the $i$ th cluster. The cluster prototypes constitute hotspots of circular areas which are shown on the map.

The Calculate reliability function calculates the reliability of each hotspot by applying the Formula (13) to assess the fuzziness of the hotspots. The reliability $R\left(A_{i}\right), i=1, \ldots, C$, assigned to the $i$ th hotspot is given by the Formula (1); it is a value in the range $[0,1]$.

Finally, the reliability thematic map was produced. Below we show the algorithm applied to extract the hotspots assessing their reliabilities (Algorithm 2).

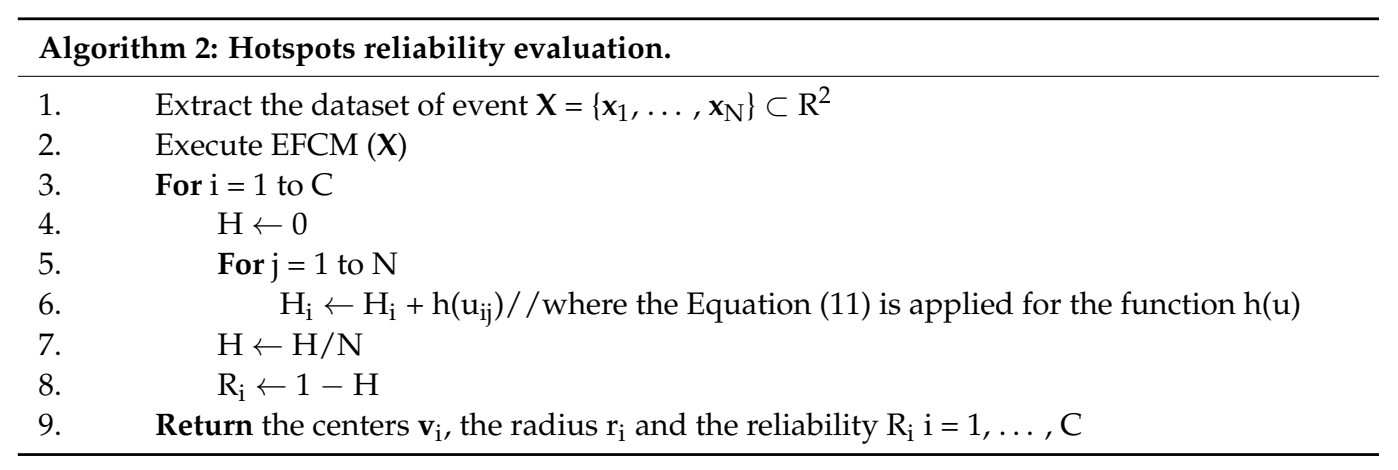

In next section we show the obtained results.

\section{Test Results}

We tested our framework on an area of study given by the district of Naples, Italy. The extension of the district is $1171 \mathrm{~km}^{2}$. The event dataset was constructed by considering the places of residence of patients who have been diagnosed with oto-laryngo-pharyngeal disease diagnosis in the last four years. These data were collected by entering only nonsensitive information and transmitted by hospitals and medical facilities. An address locator geocoding function was used to geo-refer the data points.

The event dataset is made from about 4000 data points. The GIS framework was constructed using the tool GIS Esri ArcGis Desktop 10.8; the EFCM algorithm was implemented in the GIS platform using Python libraries.

After executing EFCM, 24 hotspots were detected and plotted as circles on the map.

The thematic map with the hotspots is shown in Figure 2.

The detected hotspots have an extension between 0.6 and about $9 \mathrm{~km}^{2}$. The area and the reliability of each hotspots are shown in Table 1.

Figure 3 shows a plot graph in which the reliability dependency on hotspots area is analyzed.

Figure 3 shows that there is no linear dependency between the area of hotspots and their reliability; the very low value of the coefficient of determination $\mathrm{R}^{2}(=0.128)$ means that the smaller and more compact hotspots do not necessarily have greater reliability.

In Figure 4, the graph analyzes the linear dependency of reliability on the standard deviation. The graph shows the presence of a linear relationship between standard deviation and reliability with a mean-high value of the coefficient of determination $R^{2}(=0.864)$ : this result means that, on average, the greater the fluctuation of the values of the degrees of 
belonging of the data points to the hotspot, the greater the fuzzy entropy of the hotspot, therefore the lower its reliability.

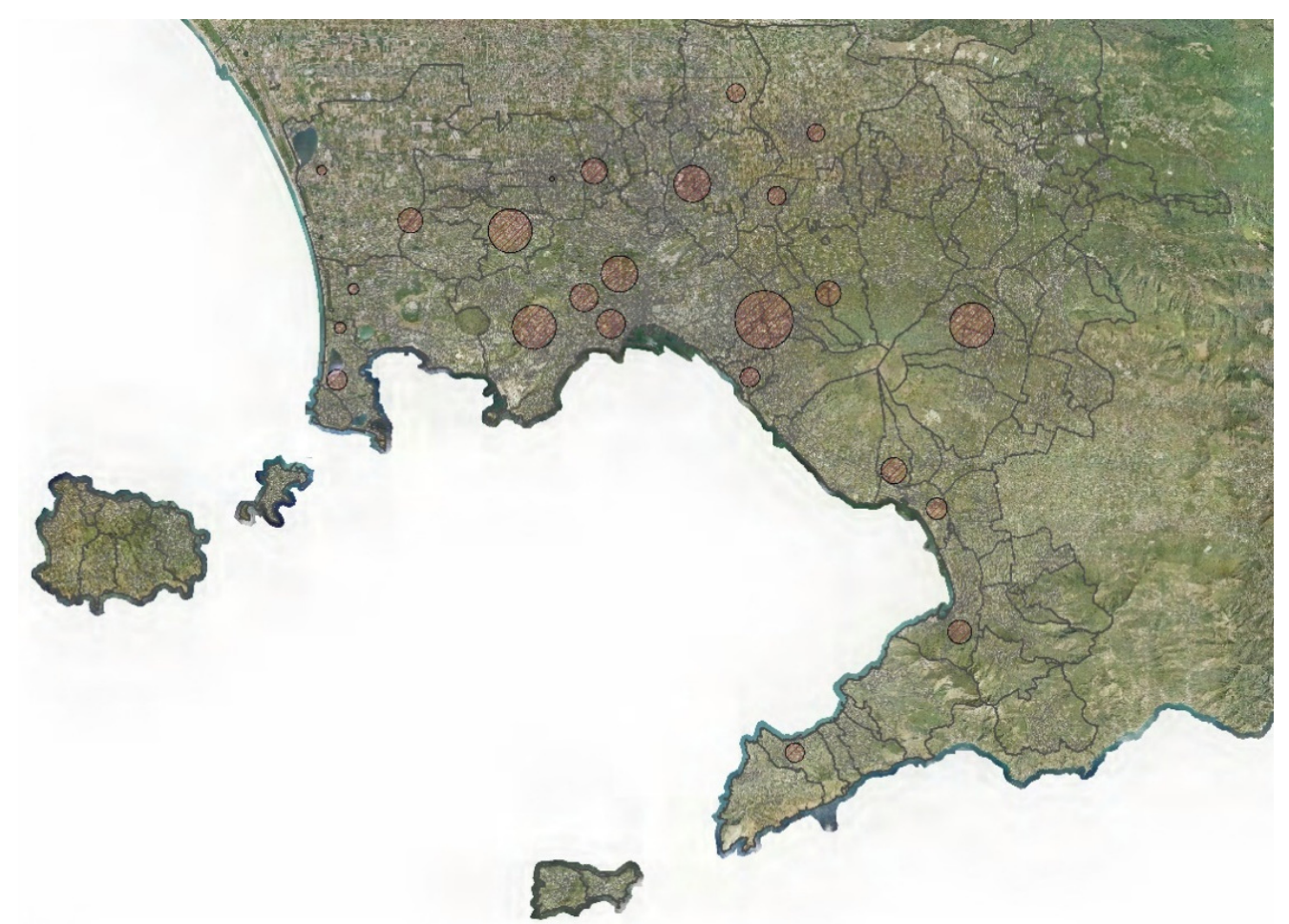

Figure 2. Thematic map with the detected hotspots.

Table 1. Area and reliability of the detected hotspots.

\begin{tabular}{cccc}
\hline ID & Area $\mathbf{( k m}^{\mathbf{2}} \mathbf{~}$ & Standard dev. & Reliability \\
\hline 01 & 0.06 & 0.045 & 0.47 \\
02 & 0.24 & 0.028 & 0.69 \\
03 & 0.31 & 0.031 & 0.68 \\
04 & 0.34 & 0.033 & 0.63 \\
05 & 0.81 & 0.033 & 0.68 \\
06 & 0.89 & 0.025 & 0.81 \\
07 & 0.92 & 0.032 & 0.67 \\
08 & 0.97 & 0.024 & 0.82 \\
09 & 1.00 & 0.022 & 0.89 \\
10 & 1.08 & 0.033 & 0.57 \\
11 & 1.18 & 0.050 & 0.41 \\
12 & 1.47 & 0.026 & 0.76 \\
13 & 1.59 & 0.034 & 0.57 \\
14 & 1.66 & 0.044 & 0.42 \\
15 & 1.76 & 0.033 & 0.66 \\
16 & 1.79 & 0.042 & 0.43 \\
17 & 2.10 & 0.035 & 0.53 \\
18 & 2.14 & 0.039 & 0.47 \\
19 & 3.51 & 0.030 & 0.57 \\
20 & 3.52 & 0.048 & 0.46 \\
21 & 5.03 & 0.045 & 0.43 \\
22 & 5.07 & 0.037 & 0.58 \\
23 & 5.30 & 0.035 & 0.61 \\
24 & 8.91 & 0.043 & 0.50 \\
\hline
\end{tabular}




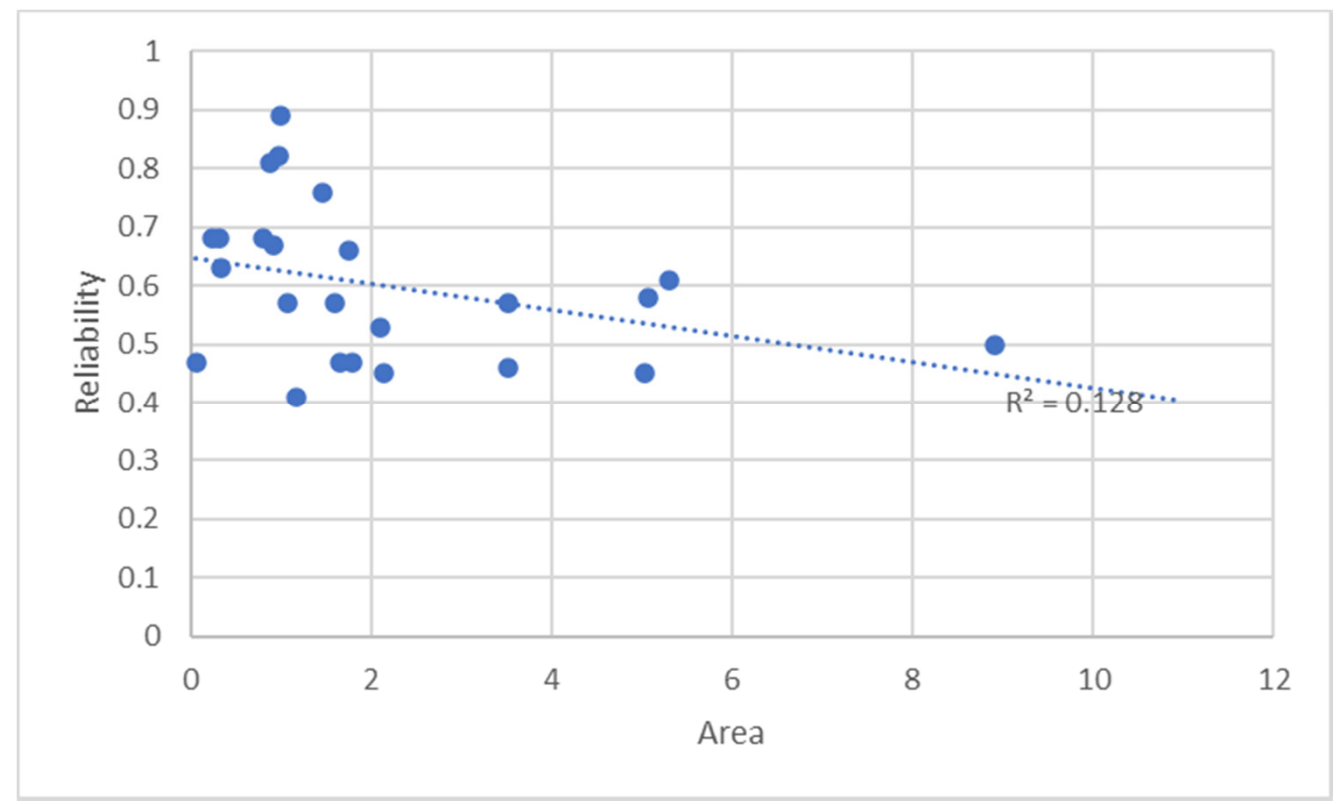

Figure 3. Linear dependency analysis results of the reliability on the area of the hotspots.

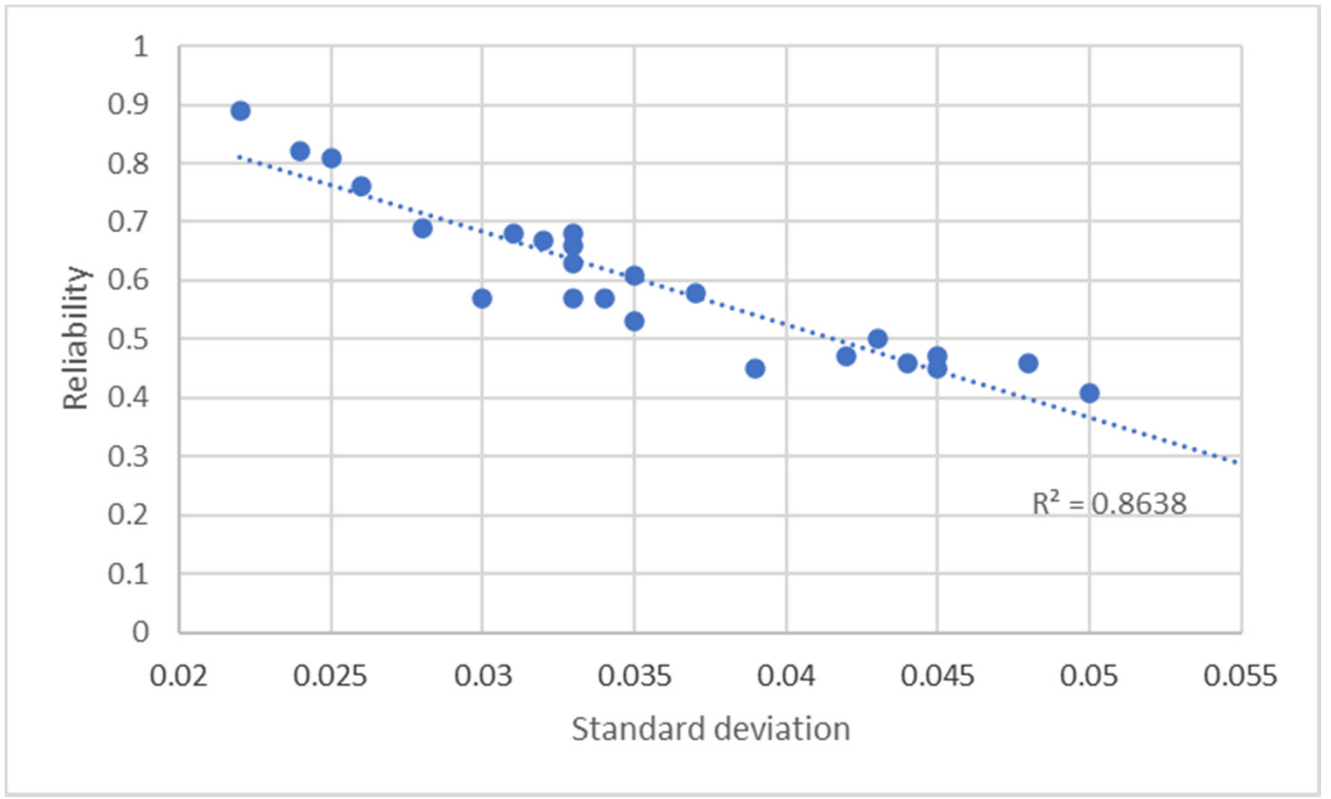

Figure 4. Linear dependency analysis results of the reliability on the standard deviation.

Figure 5 shows a thematic map of the hotspots in which three thematic classes are used, obtained by applying the Jenks Natural Breaks Classification method [30]:

- $\quad$ Low, which includes hotspots with reliability less than 0.45

- $\quad$ Mean, which includes hotspots with reliability between 0.45 and 0.6

- High, which includes hotspots with reliability greater than 0.6

This can provide information on the distribution in the study area of hotspots with different reliabilities. Hotspots with low reliability can be interpreted as hotspots in which there is greater uncertainty regarding their location and extent; on the contrary, hotspots with high reliability are hotspots whose location and extent have been detected with greater certainty. 


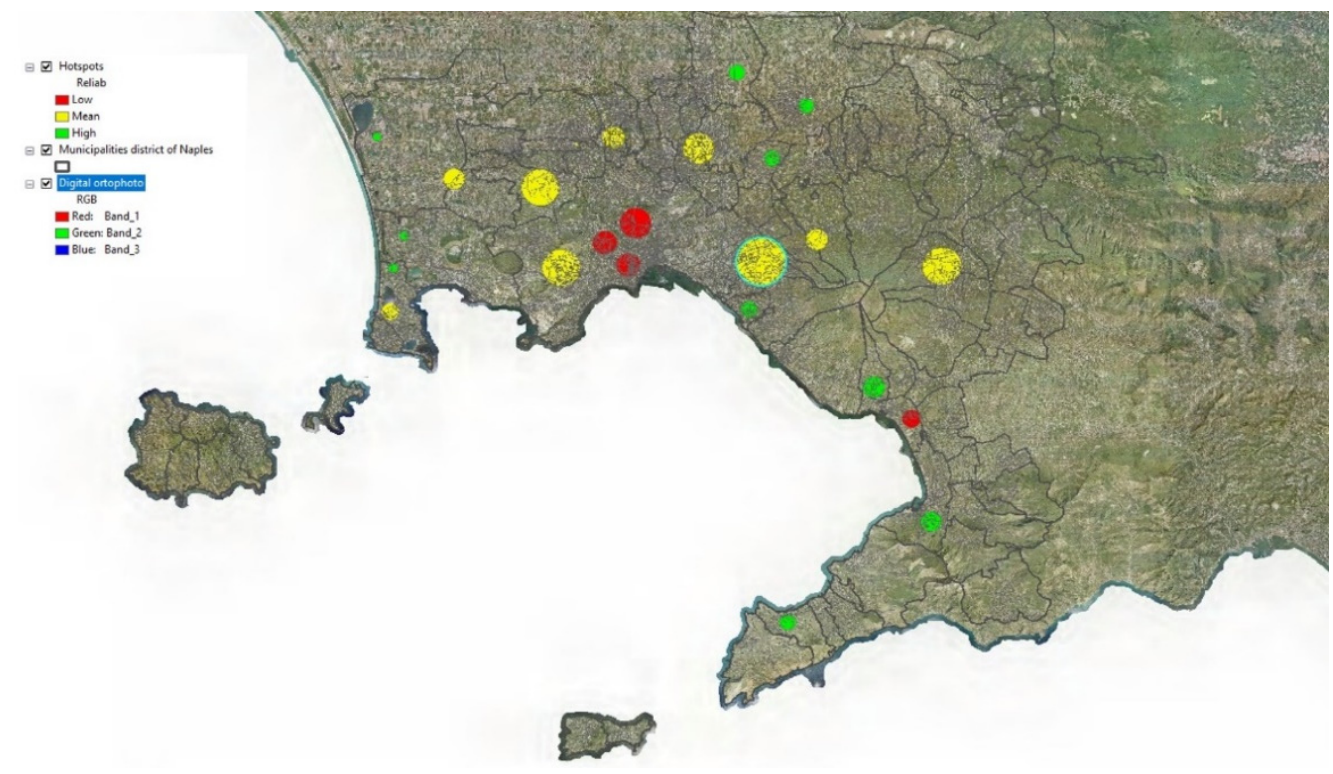

Figure 5. Thematic map with the detected hotspots.

The map in Figure 5 shows a concentration of hotspots with low reliability within the municipality of Naples; they are located in an area corresponding to the historic center of the city.

We asked a team of expert doctors who analyzed the dataset of the locations of patients diagnosed with the disease to evaluate how accurate the location and width of each hotspot detected on the map was, assigning one of the three labels: Low, Mean, and High, to each hotspot. In Table 2, the evaluations of the experts are compared with the results obtained in the thematic map in Figure 5.

Table 2. Hotspot reliability Comparison results.

\begin{tabular}{cccc}
\hline ID & Reliability & Reliability Class & Expert Evaluation \\
\hline 01 & 0.47 & Mean & Mean \\
02 & 0.69 & High & High \\
03 & 0.68 & High & High \\
04 & 0.63 & High & Mean \\
05 & 0.68 & High & Mean \\
06 & 0.81 & High & High \\
07 & 0.67 & High & Mean \\
08 & 0.82 & High & High \\
09 & 0.89 & High & High \\
10 & 0.57 & Mean & Mean \\
11 & 0.41 & Low & Low \\
12 & 0.76 & High & High \\
13 & 0.57 & Mean & High \\
14 & 0.43 & Low & Mow \\
15 & 0.66 & High & Low \\
16 & 0.42 & Low & Mean \\
17 & 0.53 & Mean & Mean \\
18 & 0.47 & Mean & Mean \\
19 & 0.57 & Mean & Mean \\
20 & 0.46 & Mean & Low \\
21 & 0.43 & Low & Mean \\
22 & 0.58 & Mean & High \\
23 & 0.61 & Mean & Mean \\
24 & 0.50 & Mean & \\
\hline & & & \\
& & &
\end{tabular}


The results obtained are correlated with the deductions made by the team of experts. The four hotspots rated with reliability Low, are also evaluated with Low reliability by the pool of experts, who found it more difficult to locate disease strains in the areas including the historic center of the municipality of Naples, due mainly to a high population density, on average homogeneous to the entire area of the historic city center.

\section{Final Considerations}

To assess the reliability of the hotspots detected in spatial analysis, we propose a framework in which the EFCM hotspot detection algorithm is used to detect the hotspots, and the De Luca and Termini's fuzzy entropy is applied to measure the reliability of the detected hotspots.

We tested our framework in a disease analysis problem; the results show that the presence of an approximately linear dependence between the reliability of the detected hotspots and the fluctuation of the membership degrees of the event data points to the corresponding fuzzy clusters. Furthermore, the spatial distribution of the reliability of the detected hotspots corresponds to the assessments made by the pool of experts.

In the future, we intend to adapt and apply our framework on massive event datasets, and to test the proposed method for measuring the reliability of predictions of future locations and displacements of hotspots.

Author Contributions: Conceptualization, F.D.M. and S.S.; methodology, F.D.M. and S.S.; software, F.D.M. and S.S.; validation, F.D.M. and S.S.; formal analysis, F.D.M. and S.S.; investigation, F.D.M. and S.S.; resources, F.D.M. and S.S.; data curation, F.D.M. and S.S.; writing-original draft preparation, F.D.M. and S.S.; writing-review and editing, F.D.M. and S.S.; visualization, F.D.M. and S.S.; supervision, F.D.M. and S.S. All authors have read and agreed to the published version of the manuscript.

Funding: This research received no external funding.

Institutional Review Board Statement: Not applicable.

Informed Consent Statement: Not applicable.

Data Availability Statement: This study did not report any data.

Conflicts of Interest: The authors declare no conflict of interest.

\section{References}

1. MacQueen, J.B. Some Methods for Classification and Analysis of Multivariate Observations. In Proceedings of the fifth Berkeley Symposium on Mathematical Statistics and Probability; Le Cam, L.M., Neyman, J., Eds.; University of California Press: Oakland CA, USA, 1967; Volume 1, pp. 281-297.

2. Levine, N. CrimeStat: A Spatial Statistical Program for the Analysis of Crime Incidents. In Encyclopedia of GIS; Shekhar, S., Xiong, H., Zhou, X., Eds.; Springer: Berlin/Heidelberg, Germany, 2010.

3. Vadrevu, K.V.; Csiszar, I.; Ellicott, E.; Giglio, L.; Badarinath, K.V.S.; Vermote, E.; Justice, C. Hotspot Analysis of Vegetation Fires and Intensity in the Indian Region. IEEE J. Sel. Top. Appl. Earth Obs. Remote Sens. 2013, 6, 224-228. [CrossRef]

4. Hajela, G.; Chawla, M.; Rasool, A. A Clustering Based Hotspot Identification Approach For Crime Prediction. Procedia Comput. Sci. 2020, 167, 1462-1470. [CrossRef]

5. Khairani, N.A.; Sutoyo, E. Application of K-Means Clustering Algorithm for Determination of Fire-Prone Areas Utilizing Hotspots in West Kalimantan Province. Int. J. Adv. Data Inf. Syst. 2020, 1, 9-16. [CrossRef]

6. Kaufman, L.; Rousseeuw, P.J. Finding Groups in Data: An Introduction to Cluster Analysis, 2nd ed.; John Wiley \& Sons: Hoboken, NJ, USA, 2005; 342p, ISBN 978-0471735786.

7. Tabarej, M.S.; Minz, S. Rough-Set Based Hotspot Detection in Spatial Data. In Advances in Computing and Data Sciences. ICACDS 2019. Communications in Computer and Information Science; Singh, M., Gupta, P., Tyagi, V., Flusser, J., Ören, T., Kashyap, R., Eds.; Springer: Singapore, 2019; Volume 1046.

8. Bezdek, J.C. Pattern Recognition with Fuzzy Objective Function Algorithms; Plenum Press: New York, NY, USA, 1981; 272p, ISBN 978-0306406713.

9. Bezdek, J.C.; Ehrlich, R.; Full, W. The fuzzy C-means Clustering Algorithm. Comput. Geosci. 1984, 10, 191-203. [CrossRef]

10. Bezdek, J.C.; Pal, S.K. Fuzzy Models for Pattern Recognition: Methods that Search for Structure in Data; IEEE Press: New York, NY, USA, 1992; 544p, ISBN 978-0780304222. 
11. Grubesic, T.H. On the Application of Fuzzy Clustering for Crime HotSpot Detection. J. Quant. Criminol. 2006, $22,77-105$. [CrossRef]

12. Kaur, R.; Sehera, S.S. Analyzing and Displaying of Crime Hotspots using Fuzzy Mapping Method. Int. J. Comput. Appl. 2014, 103, 25-28. [CrossRef]

13. Ansari, M.Y.; Prakash, A. Application of Spatio-temporal Fuzzy C-Means Clustering for Crime Spot Detection. Def. Sci. J. 2018, 68, 374-380. [CrossRef]

14. Win, K.N.; Chen, J.; Chen, Y.; Fournier-Viger, P. PCPD: A Parallel Crime Pattern Discovery System for Large-Scale Spatio-temporal Data Based on Fuzzy Clustering. Int. J. Fuzzy Syst. 2019, 21, 1961-1974. [CrossRef]

15. Bandyopadhyaya, R.; Mitra, S. Fuzzy Cluster-Based Method of Hotspot Detection with Limited Information. J. Transp. Saf. Secur. 2015, 7, 307-323. [CrossRef]

16. Besag, J.; Newell, J. The detection of clusters in rare diseases. J. R. Stat. Soc. A 1991, 154, 143-155. [CrossRef]

17. Devroye, L.; Rugosi, G. Combinatorial Methods in Density Estimation; Springer: Berlin/Heidelberg, Germany, 2001; 208p, ISBN 978-0387951171.

18. Chaney, S.; Ratcliffe, J. GIS and Crime Mapping Chap. 6, Identifying Crime Hotspots; John Wiley \& Sons: Hoboken, NJ, USA, 2013; 402p, ISBN 978-0-470-86099-1.

19. Lin, Y.-P.; Chu, H.-J.; Wu, C.-F.; Chang, T.-K.; Chen, C.-Y. Hotspot Analysis of Spatial Environmental Pollutants Using Kernel Density Estimation and Geostatistical Techniques. Int. J. Environ. Res. Public Health 2011, 8, 75-88. [CrossRef] [PubMed]

20. Harirforoush, H.; Bellalite, L. A New Integrated GIS-based Analysis to Detect Hotspots: A Case Study of the City of Sherbrooke. Accid. Anal. Prev. 2019, 130, 62-74. [CrossRef] [PubMed]

21. Di Martino, F.; Sessa, S. The Extended Fuzzy C-means Algorithm for Hotspots in Spatio-temporal GIS. Expert Syst. Appl. 2011, 38, 11829-11836. [CrossRef]

22. Kaymak, U.; Setnes, M. Fuzzy Clustering with Volume Prototype and Adaptive Cluster Merging. IEEE Trans. Fuzzy Syst. 2002, 10, 705-712. [CrossRef]

23. Di Martino, F.; Sessa, S.; Barillari, E.S.; Barillari, M.S. Spatio-temporal Hotspots and Application on a Disease Analysis Case via GIS. Soft Comput. 2014, 18, 2377-2384. [CrossRef]

24. Di Martino, F.; Sessa, S.; Mele, R.; Barillari, U.E.; Barillari, M.R. WebGIS Based on Spatio-temporal Hotspots: An Application to Oto-laryngo-pharyngeal Diseases. Soft Comput. 2015, 20, 2135-2147.

25. Di Martino, F.; Pedrycz, W.; Sessa, S. Hierarchical Granular Hotspots Detection. Soft Comput. 2020, 24, 1357-1376. [CrossRef]

26. De Luca, A.; Termini, S. Entropy and energy measures of fuzzy sets. In Advances in Fuzzy Set Theory and Applications; Gupta, M.M., Ragade, R.K., Yager, R.R., Eds.; North-Holland: Amsterdam, The Netherlands, 1979; pp. 321-338.

27. De Luca, A.; Termini, S. A Definition of Non-probabilistic Entropy in the Setting of Fuzzy Sets Theory. Inf. Control 1972, 20, 301-312. [CrossRef]

28. Di Martino, F.; Sessa, S. A New Validity Index Based on Fuzzy Energy and Fuzzy Entropy Measures in Fuzzy Clustering Problems. Entropy 2020, 22, 1200. [CrossRef] [PubMed]

29. Cardone, B.; Di Martino, F. A Novel Fuzzy Entropy-Based Method to Improve the Performance of the Fuzzy C-Means Algorithm. Electronics 2020, 9, 554. [CrossRef]

30. Jenks, G.F. The Data Model Concept in Statistical Mapping. Int. Yearb. Cartogr. 1967, 7, 186-190. 